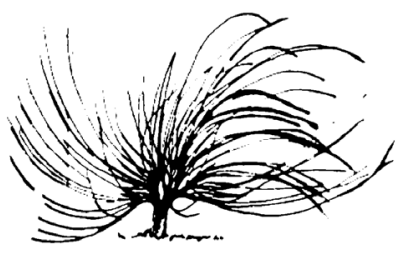

\title{
¿Por qué enseñar abstracciones en la educación primaria y secundaria? Evidencia contra las corrientes actuales
}

\author{
Martín Gonzalo Zapico ${ }^{1}$ \\ Instituto de Formación Docente Continua \\ Universidad Nacional de San Luis \\ Argentina \\ athenspierre@gmail.com
}

\begin{abstract}
Resumen
El sistema educativo argentino actualmente vive una situación que podría ser catalogada como crisis, manifiesta cuando los grupos egresados del secundario deben afrontar una carrera de nivel superior o universitaria: no tienen las competencias básicas de lectura y escritura, lo cual redunda en una alta tasa de deserción en el primer año. Este estado de situación, entre otras razones, puede atribuírsele a un enfoque que optó por dejar de lado la enseñanza directa de abstracciones, el cual postula que el aprendizaje de estas puede suceder de manera indirecta. En función de ello, proponemos una revisión de la evidencia teórica, experimental y lingüística de que la enseñanza de abstracciones y conceptos complejos es necesaria para promover no solo un mejor desempeño académico, sino también una mejor calidad de vida en general, exhibimos la urgencia de una vuelta a estas prácticas, para incentivar el desarrollo de una
\end{abstract}

Recibido: 10 de julio de 2018. Aprobado: 10 de abril de 2019.

http://dx.doi.org/10.15359/rep.14-1.1

1 Es Profesor de Letras y Filosofía recibido en la UNMDP. Actualmente se desempeña como profesor responsable en el IFDC-San Luis y doctorando en Educación en la UNSL. Ha realizado numerosas publicaciones en revistas científicas, así como tres libros y asistido a numerosas reuniones científicas en los campos de educación, lingüística y psicología. 
visión crítica de la realidad, en un mundo que tiende cada vez más a la desinformación y la sobre-simplificación. En una primera instancia se analizará evidencia teórica en torno a la importancia de las abstracciones, luego se abordará la evidencia lingüística y, por último, los trabajos de índole experimental. Los resultados del análisis sugieren que un regreso a formas de enseñanza donde las abstracciones tengan un papel primordial, permitirían mejorar el desempeño que el estudiantado tiene respecto a problemas de lectura y escritura.

Palabras clave: Educación, conceptos abstractos, enseñanza, vocabulario, educación superior.

\begin{abstract}
The argentine educational system today lives a crisis situation, which becomes evident when high school graduates must undertake a college degree: they do not have the basic skills of reading and writing, which results in a high dropout rate in their freshman year. This state of affairs can be attributed to an approach that chose to ignore the direct teaching of abstractions, postulating that they can be learned indirectly. On this basis, we propose that the direct teaching of abstractions and complex concepts is necessary to promote not only a better academic performance but a better quality of life in general. To argue this position, we will resort the opinion of teachers, experts in the field, theoretical studies and practical experience, highlighting the urgency of a return to these practices to promote the development of a critic view of reality in a world that tends increasingly leans towards disinformation and over-simplifications. In a first instance, theoretical evidence will be analyzed about the importance of abstractions; then, the linguistic evidence will be addressed and finally the experimental work will be reviewed. Evidence from the analysis suggests that a return to teaching methods where abstractions play a key role would improve the performance that students have regarding reading and writing problems.
\end{abstract}

Keywords: education, abstract concepts, teaching, vocabulary, higher education 


\section{Introducción: Crisis, estancamiento, problemas}

Hay un común acuerdo entre diversos sujetos actores (personal docente e investigador de la educación e incluso estudiantes) sobre el hecho de que en Argentina el sistema educativo se halla en una situación crítica. Sobre los asuntos que ponen de manifiesto este estado hay bastante escrito.

Tenemos, por ejemplo, los que hacen énfasis en las cada vez más escasas competencias léxicas de estudiantes en los distintos niveles (Laco, Natalia y Ávila, 2010; Valeiras, 2009), problema que se ha buscado subsanar a través de la creación de cada vez más talleres de lectura y escritura (Carlino, 2001; Carlino, 2007; Moyano, 2005) orientados más que nada a los niveles de educación superior y universitaria.

Otros estudios eligen hacer foco en los serios problemas edilicios y de infraestructura que padecen los establecimientos públicos (Bezem, 2012; Krüger, 2012), consecuencia de una progresiva segmentación de la educación pública con un interesante aumento de la matrícula privada (Bottinelli, 2013), fenómeno de naturaleza multicausal; pero de enorme impacto en la sociedad y su percepción sobre la educación pública.

Hay investigaciones que prefieren los problemas que se marcan a partir de la desacreditación del rol docente y la crisis de la autoridad tanto en los padres y madres como en las figuras institucionales (Tallone, 2012). Ante esta situación se definen dos posturas: las que sostienen que el rol docente debe mutar y conformar autoridad desde un nuevo lugar, dado que la escuela también está cambiando (Vaillant, 2010; Vezub, 2011), y las que sostienen que el rol docente está bien como está y las familias deben enseñar a sus hijos e hijas a respetar la autoridad (Mosquera, 2015; Tedesco, 1998), puesto que la obediencia a las personas mayores es algo que se aprende desde la infancia y la escuela no puede darlo.

Otros grupos de pensadores y pensadoras de la educación expresan como hipótesis que los problemas actuales en educación, más allá de sus manifestaciones particulares, tienen su origen en las desigualdades sociales de base (Dussel, 2005; Salvia, 2008) y que para lograr un verdadero cambio no se puede demandar a la escuela que haga esto o aquello, si el Estado mismo y la sociedad en su conjunto no acompañan, con acciones concretas, dicho movimiento. 
Más allá del elemento sobre el cual se apoye (el currículo, los métodos, los actores), la predilección de la mirada (cuantitativa, cualitativa o mixta) o la perspectiva sobre el fenómeno que se adopte (unos colectivos trabajan sobre las consecuencias, otros sobre las causas, otros buscan describirlo), es indudable el acuerdo que existe sobre el estancamiento al cual ha llegado el sistema educativo en el país. La escuela pública ya no está en condiciones de responder a las demandas de la sociedad, cada vez más diversas. Este asunto, que en sí mismo ya arrastra a los sistemas de educación primario y secundario, se extiende al nivel superior y universitario, donde se observa un gran problema de deserción ante de la incapacidad de hacer frente a las actividades y los textos requeridos en dichos niveles académicos, generalmente más complejos y de mayor dificultad que los trabajados en el nivel secundario.

En este contexto complejo, se hará énfasis en la problemática de las competencias léxicas fundamentales y, a su vez, se pondrá la mirada sobre uno de los temas menos o prácticamente nada abordados dentro de la línea curricular: el manejo de abstracciones.

La hipótesis que se sostiene es que el manejo de abstracciones es el primer contacto que el alumnado tiene con los elementos conceptuales más elaborados que deberá afrontar en los niveles superiores. La fuerte tendencia de los últimos veinte años ha sido (suponemos por una mala interpretación) la desaparición progresiva de los contenidos abstractos y una sobre-simplificación que impide el desarrollo de las funciones superiores necesarias para las siempre buscadas capacidades de pensamiento crítico, capacidad de reflexionar y transformar la realidad, juicios analíticos, entre otras. Los problemas de competencia léxica estarían dados, en última instancia, por una incapacidad para manejar concepciones abstractas, que redundan en complicaciones para el diseño, planificación y escritura de un texto o lectura con palabras de difícil acceso.

Para la defensa de esta hipótesis acudiremos a evidencia de naturaleza tanto teórica (sobre las abstracciones y su vinculación con la experiencia y el significado), práctica (el análisis de los modelos dominantes en la educación primaria y secundaria para tres disciplinas) como experimental (los estudios de la teoría neuro-cognitiva y el análisis de correlación entre vocabulario y desempeño académico) que permitirán evidenciar cómo el manejo del pensamiento abstracto redunda no solo en un mejor trayecto académico, sino también en mejor calidad de 
vida. Los resultados del análisis sugieren que, efectivamente, un mayor énfasis en la enseñanza de contenidos que impliquen el manejo de conceptos abstractos permitiría un mejor desarrollo de las habilidades necesarias para afrontar la educación superior.

\section{El significado, las abstracciones y su función cognoscitiva}

Indudablemente, hablar de abstracciones nos obliga a hablar, en alguna medida, del significado. Tema infinitamente prolífero, ha merecido un tratamiento desde la Antigüedad Grecolatina hasta nuestros días. Ha sido abordado, por ejemplo, desde la lingüística (Benveniste, 1966; Hjemslev, 1943; Lyons, 1980; Saussure, 1916), la semiótica (Margariños de Morentin, 1983; Morris, 1936; Peirce, 1958), la antropología (Mead, 1928), la teoría estética (Mukarosvky, 1936) y se podrían enumerar decenas más. Una revisión de las distintas ideas sobre el significado que fueron apareciendo durante el siglo XX puede estudiarse en Zapico (2017a) que realiza un recuento minucioso de cada disciplina y observa su interacción con otras problemáticas.

Para enfocar el tema que nos atañe en este escrito y enfocar la naturaleza de la abstracción en contraposición a la concreción bastará tomar de Lyons (1980) la distinción de tres grandes tipos de significados a partir de la relación lenguaje-referente para elaborar una pequeña conceptualización sobre los tipos de conceptos.

El primer grupo de conceptos pertenece a aquellos cuya referencia puede encontrarse en el mundo real. Por ejemplo: mesa, gato, árbol, televisión. Su característica más visible es la posibilidad de señalarlos. Son los llamados sustantivos concretos y su enseñanza suele establecerse en los últimos años de jardín y los primeros de la primaria.

El segundo grupo de conceptos pertenece a aquellos cuya referencia está acompañada por un concepto del primer grupo pero dicha referencia no existe en el mundo real. Por ejemplo: bien, mal, felicidad, soledad. Son los típicamente llamados sustantivos abstractos, y tienen la peculiaridad de que, si bien su referencia no existe en el mundo real, las personas los aprenden y consolidan e incluso emplean generalmente acompañando a un concepto del primer grupo. Puede que no podamos señalar al bien como entidad concreta, pero somos capaces de señalar elementos concretos que poseen relación con él, por eso se los emplea en calidad de adjetivo. Estos suelen empezar a ser enseñados desde el 
tercer grado de primaria (a partir de los ocho años los niños son capaces de manipular abstracciones) y su enseñanza se extenderá principalmente durante la secundaria.

Finalmente, y manifestando un grado absoluto de abstracción, están los conceptos abstractos cuya referencia son otros conceptos abstractos y carecen de referencia en el mundo real. Este tipo de conceptos requieren un mayor grado de manejo en la actividad académica. Palabras como, por ejemplo: paradigma, proposición, entidad, sistema, son palabras cuyas definiciones son, precisamente, conceptos abstractos y no tienen referencia alguna con la realidad material.

Entre estos tres tipos de conceptos hay relaciones de subsumisión para su aprendizaje. Es decir, para entender un concepto de segundo nivel es necesario dominar el primero, así como para entender un concepto del tercer nivel es necesario dominar el segundo nivel. La referencia de los conceptos ha sido señalada como un aspecto clave tanto en la interpretación como producción de textos académicos (García, Marta, Hall y Marín, 2005), dado que para entender, por ejemplo, su tema o su hipótesis, es necesario realizar operaciones mentales de gran nivel de abstracción. La gran problemática estaría dada, según García, Marta, Hall y Marín (2005), por las nominalizaciones (esos conceptos del tercer nivel) a las cuales el alumnado no es capaz de atribuir referencia alguna, ni siquiera en un plano netamente abstracto.

La abstracción, además, está involucrada en prácticamente todos los estilos de aprendizaje estudiados y tipificados (Bitran, Zúñiga, Lafuente, Viviani y Mena, 2003). No hay caso alguno, incluso en los que el aprendizaje es un saber hacer, en donde no haya un procesamiento categorial de la actividad. La generación de esquemas y diseños conceptuales es un proceso inherente a la actividad lingüística de cualquier naturaleza. Basta ir al día a día y revisar nuestros propios diálogos para observar la gran cantidad de abstracciones que empleamos, incluso sin darnos cuenta. Hay que recordar, a propósito de esto, lo que muchas teorías de la psicología y el aprendizaje denominan realidad psíquica (Aebli, 1988), que consiste en un esquema abstracto que le permite a los sujetos ordenar su experiencia del mundo, llena de estímulos diversos. La abstracción es de una presencia tal en la vida de los sujetos, que no puede ser desconocida en la educación, en especial la primaria y la secundaria, como un contenido fundamental, si se habla de aprendizaje significativo. 


\section{La experiencia y su relación con el aprendizaje}

En este panorama, ¿qué lugar ocupa la experiencia del mundo?, ¿cuál es el papel de los sentidos? Desde la psicología y la neurología (Estévez, García y Barraquer, 1997) encontramos una doble dimensión del entendimiento de la experiencia. Una interna y relacionada con el aprendizaje que el cerebro realiza como proceso de resignificación que, a su vez, requiere una experiencia externa de intercambio de estímulos e información con el medio. La experiencia en este sentido se relaciona con la interacción individuo-ambiente, donde acontece un proceso de retroalimentación constante. En líneas similares está la biología (Castorina, 1972), que tiende a asimilar la noción de experiencia con la estimulación de neurorreceptores que posibilitan la adquisición de distintos conocimientos, los cuales, a su vez, serán reacomodados y reestructurados.

En este mismo esquema general (de base constructivista) encuadran su pensamiento otros teóricos prominentes como Vigotsky, Luria y Ausubel (Huber, 2008). Estos optan por enmarcar sus análisis sobre el aprendizaje en la interacción que el sujeto tiene con el mundo, tanto interior (su realidad psíquica) como exterior (la realidad social). En este esquema se han cimentado una gran cantidad de discursos en educación (Giménez, Téllez, y Sierra, 2009; Giroux y MacLaren, 2003; Skliar, 2002; Martínez-Bonafé y Adél, 2003) que, hoy día y durante dos décadas, han sostenido la necesidad de acercar la experiencia del mundo a la escuela.

Este modelo de enseñanza, que en su momento se erigió para criticar la absoluta disociación observada entre los contenidos enseñados en la escuela y la vida de los sujetos, parece haber perdido el rumbo al punto de postular que los contenidos de naturaleza abstracta (y que lógicamente es difícil reponer sus referentes en la realidad) debían reducirse al mínimo de los currículos escolares en Argentina e incluso eliminarse (Oliva-Figueroa, 2010; Smith, 2004) La interpretación radical de las teorías contextualmente situadas del aprendizaje, sostenemos, sucedió como un posible error de interpretación o falta de conciencia crítica a la hora de adscribirse a las teorías de moda que proliferaban en los noventa.

A continuación revisaremos el contexto histórico en el cual llegan estas nuevas teorías y cómo su interpretación se vio reflejada en políticas concretas sobre el currículo en distintas disciplinas. 


\section{Evidencia práctica: Modelos disciplinares}

Los mediados de los noventa vieron proliferar, a partir del marco epistemológico descrito anteriormente, aplicaciones concretas en diversas disciplinas que, de forma más o menos sistemática, fueron aplicadas en las planificaciones de diversos grupos docentes y programas. Vamos a tomar, en el marco de la educación secundaria y primaria, el modelo realista en educación matemática, el modelo comunicativo en lengua y el modelo sociohistórico revisionista en historia y a revisar sus postulados para observar cuáles son sus propuestas concretas sobre la enseñanza e hipotéticos aprendizajes. La elección de estas tres asignaturas está definida por ser aquellas que más carga horaria tienen de acuerdo con lo estipulado por lineamientos nacionales, así como ser tres materias que suelen evaluarse en las pruebas estandarizadas tanto internacionales como nacionales.

Marín (2003), en una revisión, se refiere al enfoque comunicativo de la lengua como aquel en el cual predomina la situación comunicativa como situación de excelencia para el aprendizaje de la lengua. Es decir, dado que el lenguaje es una herramienta que utilizamos en la comunicación, es a través de ella que se puede llegar a una adquisición significativa de la lengua, a través de las prácticas del lenguaje que se ponen en juego en el día a día. Este modelo, señala la autora, vendría a superar el viejo modelo tecnocrático de la enseñanza de la lengua, en el cual el foco estaba puesto en el aprendizaje de abstracciones sin relación alguna con la realidad del alumnado, tales como la gramática en abstracto, el análisis oracional, el uso del diccionario para la adquisición de vocabulario, entre otros. Contra esos preceptos, supuestamente obsoletos, se contraponen la llegada a la gramática basándose en el análisis y reflexión del uso, sacar completamente de los planes de estudio el análisis oracional, desprenderse del diccionario y promover el conocimiento del significado en función del contexto, etc. Finalmente, se habla también de la incorporación de una pluralidad de prácticas. Si antes se privilegiaba el texto escrito de manera casi privativa, en este nuevo enfoque se tratan, de manera explícita, la oralidad y la expresión como contenidos concretos.

Alsina (2007), en su trabajo sobre el modelo cotidiano de aproximación realista en las matemáticas, define este nuevo modelo como aquel en el cual predomina el empleo de la matemática como mediador fundamental entre el sujeto y su experiencia de vida. Es decir, en vez 
de demandar al alumnado que busque las matemáticas en la vida real a través de planteos que en gran medida le exceden, se busca poner de manifiesto que la matemática es parte fundamental del día a día y, por ende, puede ser usada para el análisis y resolución de problemas reales amparados en el sentido común. Este modelo busca alejarse de lo que el propio autor denuncia como una disociación muy grande y grave entre los espacios de sentido que proponen los ejercicios típicos de los manuales de matemática históricos, a los que acusa de generar realidades falsas o ridículas, a veces ilógicas. Propone, en cambio, una serie de ejemplos concretos en los cuales él como docente de matemática puede relacionar la experiencia del mundo, las emociones y una gran cantidad de situaciones concretas con matemáticas tanto simples como complejas.

En historia se han propuesto modelos que cambian de manera radical el objeto de estudio de la historia en su enseñanza así como la misma metodología de abordaje. La gran historia, la historia de la civilización occidental y las jerarquías históricas seccionadas (historia mundial, latinoamericana, argentina) es reemplazada por una visión que privilegia la denominada microhistoria o historia cultural. A su vez, con gran influencia del revisionismo histórico, se propone un enfoque de naturaleza hermenéutico-interpretativo que aspira a la comprensión holística y sintética de un momento histórico en contraposición al conocimiento concreto de sucesos anclados en datos concretos como fechas, nombres, sucesos. La perspectiva pasa del hecho aislado al contexto, del momento al proceso. Este cambio de rumbo está fundamentado tanto en el poco interés que supuestamente lograba la disciplina en la primaria y secundaria como con la poca capacidad de traslado a la realidad que posibilitaban los contenidos por su propia descontextualización.

Algo que comparten en común los tres modelos al observarlos someramente es la buena voluntad que tienen respecto a la enseñanza de sus disciplinas. A primera vista, y en el plano teórico, sus postulados son completamente coherentes y necesarios. Es lógico que para llevar a cabo procedimientos, razonamientos y operaciones complejas con el saber, es necesario partir de bases concretas. Y además es lógico y esperable que a la hora de abordar abstracciones tales como, por ejemplo, la categoría de objeto directo en lengua, la noción de función en matemática o la idea de revolución en historia, se busque tomar como referentes eventos y sucesos que el alumnado pueda relacionar con su propia experiencia. Es también necesario que los métodos de abordaje cambien. 
Si esperamos que las disciplinas colaboren con el objetivo enunciado en todos los documentos oficiales de generar sujetos críticos, el siempre presente "estudiar de memoria" queda naturalmente desechado puesto que no promueve nada más que la mera capacidad de almacenamiento y repetición de datos de forma acrítica e irreflexiva.

Ahora, ¿por qué estas formas de enseñanza no parecen haber dado resultado? Los testimonios de personal docente e investigador, e incluso estudiantes que acceden a su primer año en carreras terciarias/ universitarias (Beltran y La Serna Studzynsky, 2015; Torres-Morillo y Bethencourt, 2013) dan cuenta de una insuficiencia para manipular ciertas abstracciones, que a su vez redundan en las complicaciones tanto en la interpretación como producción de textos académicos de diversos formatos. Esto, postulamos, pudo ser debido a la falta de profundidad con que se abordaron estas propuestas interesantes. Es decir, la interpretación reduccionista de los enunciados de dichos modelos da como resultado esperable una descomplejización de los contenidos, puesto que se alejan en gran medida de la experiencia de vida estudiantil. Si retomamos lo analizado respecto a los tipos de significado y de conceptos, resulta obvio que aquellos que tengan una referencia nula en las realidades de los sujetos tenderán a desaparecer de los planes de estudios por ser considerados innecesarios.

Es lo que sucedió efectivamente. En el caso de lengua y literatura (Zapico, 2017b) se puede observar la desaparición progresiva de la gramática y el análisis oracional. En la matemática (González, 2018) se ha producido una simplificación peligrosa de la dificultad y profundidad con ciertos constructos como las ecuaciones, que son progresivamente subidas de grado para su inserción en el currículo. En historia (Carretero, 2017) se ha dejado de lado completamente el método memorístico de aprendizaje y se ha propuesto una forma de abordaje completamente cualitativa. ¿Pero que grado de utilidad real representan estos giros didácticos para el alumnado?

\section{Evidencia experimental a favor de las abstracciones}

Irónicamente, los estudios en neurología muestran que el aprendizaje de nociones matemáticas complejas en jóvenes les permite no solo obtener un mejor desempeño en carreras que efectivamente tienen matemática, sino que mejora de manera indirecta sus habilidades 
de cálculo y probabilidad, empleados diariamente en la vida cotidiana (Martínez, 2012). Estudios en pedagogía (Batanero 2006; Font, 2006; Fuentes, 2008) ponen de manifiesto la mejora que se da en poblaciones expuestas a una educación matemática compleja respecto a la resolución de problemas del tipo vida cotidiana; en contraste con otra población de igual edad, pero distintas escolarización matemática, lo cual sugiere que el aprendizaje de abstracciones complejas permite la resignificación de otras más simples, pues desarrollan, así, una red de sentido. Además, se ha demostrado que el contacto con la matemática permite que niños y niñas con Síndrome de Down obtengan notorias mejorías en sus capacidades operacionales abstractas (Ortega-Tudela y Gómez-Ariza, 2007), lo cual indica que en la población infantil estándar es necesaria la estimulación temprana y el contacto con abstracciones para mejorar su autonomía respecto al manejo de nociones no concretas.

En lengua y literatura tenemos evidencia en tres planos. En un plano teórico se puede encontrar apoyo en el campo de las neurociencias. Específicamente la teoría neuro-cognitiva de Lamb $(1999,2004)$ que, construyendo hipótesis y enunciados sobre el sistema lingüístico del individuo a partir de los conocimientos que se tienen sobre el cerebro y su relación con el lenguaje, postula que la lengua no es sino un gran conjunto de redes relacionales, donde el "significado" de un lexema no es más que todos aquellos significados que activa su estimulación. Como señala Gil (2009), hay un correlato muy interesante entre esta forma de abordar el lenguaje y las implicancias pedagógicas que de ella se derivan. Si estamos ante un conjunto de redes relacionales que se comportan de manera análoga a neuronas, hay fundamentalmente tres procesos posibles para el desarrollo de la red:

- Formación de nuevas conexiones entre nexiones: Posibles relaciones que estaban latentes a partir de un estímulo fuerte empiezan a cobrar actividad.

- Cambio en la fuerza de las conexiones: A través del uso o desuso que se haga de las conexiones, estas podrán o aumentar su fuerza o disminuir.

- Cambio en las funciones de umbral: Se define umbral como la mínima cantidad de estímulo que debe recibir una conexión para ser estimulada, así, una conexión que es estimulada frecuentemente pasa a tener un umbral mucho menor que otra que solo es estimulada de manera escasa. 
En función de estas posibilidades, la ampliación del vocabulario, su mero aumento cuantitativo, tiene consecuencias positivas para el sistema lingüístico del individuo, dado que amplía enormemente su capacidad no solo de interpretar, sino también de producir enunciados.

En experiencias prácticas, en especial en lengua inglesa, hay una gran variedad de estudios que ponen de manifiesto cómo la enseñanza directa de vocabulario contribuye al desarrollo de la lengua (una revisión del tema y el sistema particular de bits de idiomas puede ser visto en Navarro-Cordon, 2002). Esta postura se deja ver, en especial, en todos los planes de profesorado de inglés en Argentina, donde en cada una las materias (incluso en las gramáticas) hay una parte destinada al trabajo con "palabras clave" y "vocabulario específico" de una determinada unidad temática. Esta lógica rige también para los libros de enseñanza del inglés de todos los niveles, que tienen para cada sección un espacio destinado al trabajo intensivo con el vocabulario a través de métodos de enseñanza directa.

En investigaciones científicas, especialmente en el área de desempeño académico, los trabajos de Zapico (2016a, 2016b) ponen de manifiesto una relación en principio correlacional entre el vocabulario léxico-productivo y el desempeño académico en el nivel superior. Si se encontraran correlaciones significativamente estadísticas en futuros estudios, esto daría paso directo a la necesidad de desarrollar en las instancias previas al nivel superior (nivel primario y secundario) un desarrollo del léxico-productivo con métodos de enseñanza directa. Incluso con el dato de que existe una correlación entre ambas, el vocabulario no puede seguir siendo dejado de lado en los programas y planificaciones de niveles primario y secundario.

Curiosamente, por el lado de la memorización, los estudios parecen reconocer beneficios no solo como método en sí mismo sino también en el ámbito educativo. En un nivel amplio, la realización de tareas de memorización voluntaria, con auxiliares mnemotécnicos o sin estos, permite una ampliación de la base léxica de forma transversal al contenido (Moreno-Cuellar, 2013). Es decir, al forzar al cerebro a recordar datos, estos se incorporan en la red semántica de los sujetos sin necesidad de un procesamiento activo, por la mera coocurrencia y aparición sistemática. La tesis de licenciatura de Cedillo-Mantilla (2012) pone de manifiesto la importancia de la implementación de la denominada gimnasia cerebral (ejercicios varios entre los que está la 
memorización) para la mejora del desempeño de niños y niñas con dificultades de aprendizaje. En el ámbito propio de la historia y su enseñanza, más de una defensa del modelo de enseñanza interpretativo de la historia reconoce la necesidad de la memorización y comprensión de la historia antes de su posible crítica y contrapunto (Carrasco, Molina y Puche, 2014; Cruz, 2007). Incluso hay estudios metodológicos en la disciplina (Salamanca-Ávila y Salamanca-Ávila, 2012) que ponen de manifiesto los beneficios que da la memorización cuando se trabaja de forma simultánea con otros métodos.

Como se ha observado, en todos los casos el empleo de abstracciones complejas en la enseñanza o incluso métodos memorísticos, ambos desdeñados por una interpretación sesgada de las nuevas corrientes educativas, trae beneficios tanto a corto como largo plazo, dentro y fuera del ámbito académico, para el estudiantado.

\section{Conclusiones}

Hemos revisado, tanto desde una perspectiva general como específica, el rol que pueden tener las abstracciones como constructos teóricos a la hora de promover el aprendizaje. En primera instancia, se ha analizado el concepto de abstracción y cómo su vinculación con el concepto de experiencia en educación es fundamental, puesto que no se puede llegar a lo abstracto sin pasar por lo concreto. Luego, se ha analizado cómo lo abstracto está íntimamente relacionado con la constitución del significado lingüístico, pues este es el medio a través del cual accedemos al conocimiento del mundo. Finalmente, se ha revisado la evidencia empírica tanto en lo relativo a los currículos vigentes en los últimos años, así como en experimentos. Se ha partido de un estado de situación ya denunciado como preocupante para tratar de desentrañar una de las posibles causas de dicha condición. En el análisis se han contrapuesto dos formas de entender el aprendizaje, las cuales, por su misma conformación, son complementarias; pero han sido interpretadas de forma incorrecta al parecer.

El error de buscar que lo aprendido tenga que ver con el mundo real ha desplazado los conceptos abstractos de la enseñanza y, sin querer eso, ha provocado una reducción de la experiencia educativa. Ya se ha estudiado la incidencia que tiene el lenguaje en la forma de abordar y comprender la realidad. Disminuir la cantidad de abstracciones 
manejadas por estudiantes no solo redunda en un deterioro de sus capacidades académicas sino también de su calidad de vida. Estamos, sin querer, quitando herramientas a los grupos de jóvenes para que puedan afrontar un mundo cada vez más cambiante, complejo y competitivo.

Hacemos creer al alumnado que los contenidos de la secundaria se reducen a lo que ya conocen o han vivido, cuando tienen en su futuro un sin fin de experiencias por vivenciar. ¿Y cómo esperamos que tengan las herramientas para comunicarse, expresarse y hasta resignificar sus sentires, si con el argumento de acercar la escuela a la vida les quitamos recursos?

Es necesaria una toma de conciencia respecto a lo que estamos haciendo como comunidad científica y educativa con los contenidos y los métodos. No podemos ignorar las evidencias que ponen de manifiesto la utilidad de ciertos constructos y métodos que, por ya tildados de viejos, tendemos a descartar apriorísticamente. No es que las corrientes nuevas hayan traído ideas malas o infructíferas, todo lo contrario: son nuestras generaciones quienes no las hemos interpretado correctamente. Nuestras leyes y documentos educativos de cabecera nos exigen como deber formar sujetos críticos de su realidad, capaces de pensar y pensarse a si mismos para cambiar su entorno. ¿Cómo esperamos lograr eso sin un sólido manejo conceptual, articulado por conceptos abstractos? ¿Cómo hacer análisis del discurso y develar la intencionalidad del lenguaje, sin categorías como sustantivo, objeto? ¿Cómo realizar una revisión exhaustiva y crítica de un suceso histórico aplicando la noción de perspectiva si no se pueden ubicar las coordenadas precisas de dicho acontecimiento?

La crisis actual del sistema educativo es indudablemente un fenómeno multicausal. No obstante, eso no es motivo suficiente para bajar los brazos. Cada persona debe hacer su aporte a conciencia desde su área de trabajo. Docentes deben revisar sus prácticas y actualizarse a fines de no utilizar información errónea, personal investigador debe avocar sus esfuerzos en los problemas concretos que tiene el sistema y posibles formas de subsanarlo. Incluso las familias y el propio alumnado tienen responsabilidad, en este fenómeno siempre cambiante llamado educación. 


\section{Referencias}

Aebli, H. (1988). Doce formas básicas de enseñar: una didáctica basada en la psicología (Vol. 50). Madrid: Narcea Ediciones.

Alsina, C. (2007). Si Enrique VIII tuvo 6 esposas, ¿cuántas tuvo Enrique IV? El realismo en educación matemática y sus implicaciones docentes. Revista Iberoamericana de educación, 43, 85-101.

Batanero, C. (2006). Razonamiento probabilístico en la vida cotidiana: Un desafio educativo. Investigación en el aula de matemáticas. Estadística y azar. Granada: Sociedad de Educación Matemática Thales.

Bitran, M., Zúñiga, D., Lafuente, M., Viviani, P., y Mena, B. (2003). Tipos psicológicos y estilos de aprendizaje de los estudiantes que ingresan a Medicina en la Pontificia Universidad Católica de Chile. Revista médica de Chile, 131(9), 1067-1078.

Benveniste, E. (1966). Curso de lingüistica general (Tomo 1). Madrid, España: Editorial Siglo XXI.

Beltrán, B., y La Serna Studzinski, K. (2009). ¿Cuán relevante es la educación escolar en el desempeño universitario? Santiago: Ediciones Universidad del Pacífico.

Bezem, P. (2012). Equidad en la distribución de la oferta de educación pública en Argentina. Buenos Aires: Inter-American Development Bank.

Bottinelli, L. (2013). ¿Por qué crece la educación privada. Le Monde Diplomatique, Dossier La educación en Debate, 12(1), 224-238.

Carlino, P. (2007). ¿Qué nos dicen las investigaciones internacionales sobre escritura en la universidad? Cuadernos de Psicopedagogía, 4, 21-40.

Carlino, P. (2001). Hacerse cargo de la lectura y la escritura en la enseñanza universitaria de las ciencias sociales y humanas. I Jornadas sobre La lectura y la escritura como prácticas académicas universitarias. Departamento de Educación, Universidad Nacional de Luján, Luján, Prov. de Buenos Aires.

Carrasco, C. J. G., Molina, J. O., y Puche, S. M. (2014). Aprender a pensar históricamente. Retos para la historia en el siglo XXI. Tempo e Argumento, 6(11), 05-27.

Castorina, J. A. (1972). Biología y conocimiento de Jean Piaget. Lima: Tarea Ediciones. 
Carretero, M. (2017). Cambio conceptual y enseñanza de la historia. Tarbiya, Revista de Investigación e Innovación Educativa, (26), 73-83.

Cedillo-Mantilla, M. (2012). Elaboración de un plan de intervención basado en la gimnasia cerebral para niños y niñas de 6 a 7 años de edad, con problemas de aprendizaje (Tesis para la obtención de grado). Universidad del Azuay, Ecuador.

Cruz, M. M. B. (2007). Evaluando la dinámica de sistemas como una herramienta para enseñar historia. Revista de Dinámica de Sistemas, 3(2), 3-60.

Dussel, I. (2005). Desigualdades sociales y desigualdades escolares en la Argentina de hoy. Algunas reflexiones y propuestas. En J. C. Tedesco (Comp.), ¿Cómo superar la desigualdad y la fragmentación del sistema educativo argentino? (pp. 35-85). Buenos Aires, Argentina, UNESCO.

Estévez, A., García, C., y Barraquer, L. I. (1997). La memoria y el aprendizaje: "experiencia" y "habilidad" en el cerebro. Rev Neurol, 25(148), 1976-1988.

Font, V. (2006). Problemas en un contexto cotidiano. Cuadernos de pedagogía, 355, 52-54.

García, N., Marta, M., Hall, B., y Marín, M. (2005). Ambigüedad, abstracción y polifonía del discurso académico: Interpretación de las nominalizaciones. Revista signos, 38(57), 49-60.

Gil, J. (2009) Neurología y lingüística: La teoría de las "redes relacionales" como una alternativa ante Chosmky. Revista de Investigación Lingüistica, 12, 343-374.

Giménez, A. M., Téllez, G. L., y Sierra, B. (2009). Competencias básicas: Sobre la exclusión de la competencia motriz y las aportaciones desde la educación física. Retos: nuevas tendencias en educación física, deporte y recreación, 16, 51-57.

Giroux, H. A., y MacLaren, P. L. (2003). Por uma pedagogia crítica. Territórios contestados: o currículo e os novos mapas políticos e culturais, 5, 144-158.

González, F. E. (2018). Historia de la educación matemática en Latinoamérica: 10 claves para su comprensión. UNIÓN, Revista Iberoamericana de Educación Matemática, 52, 279-305. 
Hjemslev, L. (1943). Prolegomena to a Theory of Language (Traducción al inglés por F. J. Whitfield, $2^{\text {da }}$ ed.) Madison: University of Wisconsin Press.

Huber, G. L. (2008). Aprendizaje activo y metodologías educativas. Active learning and methods of teaching. Tiempos de cambio universitario, 59, 59-82.

Krüger, N. (2012). La segmentación educativa argentina: Reflexiones desde una perspectiva micro y macrosocial. Páginas de Educación, 5(1), 137-156.

Laco, L, Natalia, L y Ávila, M (2010). La lectura y escritura en la formación académica, docente y profesional. Buenos Aires: Editorial de la Universidad Tecnológica Nacional.

Lamb, S. (1999). Senderos del cerebro. Mar del Plata: Eudem.

Lamb, S. (2004). Language and Reality. London \& New York: Continuum.

Lyons, J. (1980). Semántica. Barcelona: Teide.

Margariños de Morentin, J. (1983). El signo. Las fuentes teóricas de la semiología. Buenos Aires: Hachette.

Marín, M. (2003). Los puntos críticos de incomprensión de la lectura en los textos de estudio. Estado de la cuestión. Lectura y vida, 2(1), 1-10.

Martínez-Bonafé, J., y Adell, J. (2003). Viejas pedagogías, nuevas tecnologías. Cuadernos de pedagogía, 11, 99-101.

Martínez, N. P. S. (2012). Cálculo aplicado: Competencias matemáticas a través de contextos. Ciudad de México: Cengage Learning Editores.

Mead, G. (1928). Espíritu, persona y sociedad. Buenos Aires: Paidós.

Moreno-Cuellar, J. A. (2013). Recursos multimedia y mnemotecnia para la adquisición de vocabulario en inglés. Revista Virtual Universidad Católica del Norte, 1(38), 112-126.

Morris, C. (1936). The Concept of Meaning in Pragmatism and Logical Positivism. In Actes du Huitième Congrès International de Philosophie, Prague, Czechoslovakia, 2-7 September 1936.

Mosquera, C. E. M. (2015). Rumiar sobre el positivismo o trasformar la práctica educativa pedagógica docente. Actualidades Pedagógicas, 65, 217-228.

Moyano, E. I. (2005). Una propuesta didáctica para la enseñanza de la lectura y la escritura en lengua materna. En Moyano (Presidencia), 
I Jornadas de Enseñanza de la Lengua y la Literatura. Teorías literarias y lingüisticas en los niveles medio y superior. Buenos Aires: UBA.

Mukarosvky, J. (1936). Escritos de estética y semiótica del arte. Bogotá: Plaza y Janes Editores.

Navarro-Cordon, M. (2002). La enseñanza aprendizaje del vocabulario en inglés y la expresión escrita. Buenos Aires: Sudamericana.

Oliva-Figueroa, I. (2010). Breve cartografía de una disonancia epistémica: Educación, complejidad y reforma. Polis (Santiago), 9(25), 321-335.

Ortega-Tudela, J. M., y Gómez-Ariza, C. J. (2007). Nuevas tecnologías y aprendizaje matemático en niños con síndrome de Down: generalización para la autonomía. Pixel-Bit. Revista de medios y educación, 29, 59-72.

Peirce, C. (1958). Collected Papers. Cambridge: Harvard University Press.

Salamanca-Ávila, L., y Salamanca-Ávila, M. E. (2012). La supervisión de adultos: Uso de mapas conceptuales de ruta como herramienta para aumentar la cohesión, motivación y memorización. Malta: Valleta Editorial.

Salvia, A. (2008). Jóvenes promesas. Trabajo, educación y exclusión social de jóvenes pobres en la Argentina. Buenos Aires: Miño y Dávila.

Saussure, F. (1916). Curso de lingüística general. (Traducción, notas y prólogo de Amado Alonso). Buenos Aires: Losada.

Skliar, C. (2002). Alteridades y pedagogías. O... i y si el otro no estuviera ahí. Educação \& Sociedade, 23(79), 85-123.

Smith, R. (2004). Abstracción y finitud: educación, azar y democracia (L. Galera, trad.). Recuperado de https://www.google.com/ search?client $=$ opera \&q $=$ Smith $\% 2 \mathrm{C}+\mathrm{R} .+(2004) .+$ Abstracci $\%-$ $\mathrm{C} 3 \% \mathrm{~B} 3 \mathrm{n}+\mathrm{y}+$ finitud $\% 3 \mathrm{~A}+$ educaci $\% \mathrm{C} 3 \% \mathrm{~B} 3 \mathrm{n} \% 2 \mathrm{C}+\mathrm{azar}+\mathrm{y}+-$ democracia $+($ L. + Galera $\% 2 \mathrm{C}+$ trad. $) \&$ sourceid $=$ opera\&ie $=\mathrm{U}-$ TF-8\&oe $=$ UTF-8

Tallone, A. E. (2012). Las transformaciones de la autoridad docente, en busca de una nueva legitimidad. Cuadernos del Ministerio de Educación de la Nación. Buenos Aires: Ministerio de Educación.

Tedesco, J. C. (1998). Fortalecimiento del rol de los docentes: Balance de las discusiones de la $45^{\circ}$ sesión de la Conferencia Internacional 
de Educación. Revista latinoamericana de innovaciones educativas, 29, 15-40.

Torres-Morillo, M., y Bethencourt, M. T. (2013). Los mundos escolares narrados por los jóvenes universitarios. Paradigma, 34(1), 053-068.

Vaillant, D. (2010). La identidad docente. La importancia del profesorado. Revista Novedades Educativas, 22(234), 1-17.

Valeiras, N. (2009). El rendimiento académico y la escritura en ingresantes de carreras de exactas. Revista Educación en Biología, $12(1), 23-44$.

Vezub, L. F. (2011). La formación y el desarrollo profesional docente frente a los nuevos desafíos de la escolaridad. Profesorado. Revista de Currículum y Formación del Profesorado, 11(1), 1-23.

Zapico, G. (2017a). Sinonimia y significado: Una revisión. Barcelona: EAE.

Zapico, M. G. (2017b). Sobre la necesidad de la enseñanza directa del vocabulario en los niveles primario y secundario del sistema educativo argentino. Revista Ensayos Pedagógicos, 12(2), 33-45.

Zapico, M. G. (2016a). Evaluación de estrategias de enseñanza orientadas a competencias: Una comparación de caso entre estilo orientado a la competencia y el orientado al contenido. Revista Ensayos Pedagógicos, 11(1), 135-146.

Zapico, M. G. (2016b). Evaluación de desempeño académico: La competencia léxica como competencia con validez productiva. Revista Ensayos Pedagógicos, 11(2), 159-171. 
